\title{
Strong convergence of Krasnoselski-Mann iteration for a countable family of asymptotically nonexpansive mappings in $\mathrm{CAT}(0)$ spaces
}

\author{
Shanguang Qian ${ }^{\mathrm{a}}$, Wei-Qi Deng ${ }^{\mathrm{b}, *}$ \\ ${ }^{a}$ Architectural Engineering Faculty, Kunming Metallurgy College, Kunming, Yunnan, P. R. China. \\ ${ }^{b}$ School of Statistics and Mathematics, Yunnan University of Finance and Economics, Kunming, Yunnan, P. R. China.
}

Communicated by Y. J. Cho

\begin{abstract}
Based on a specific way of choosing the indices and a new concept, namely, an analogue of inner product, a modified Krasnoselski-Mann iteration scheme is proposed for approximating common fixed points of a countable family of asymptotically nonexpansive mappings; and a strong convergence theorem is established in the framework of CAT $(0)$ spaces. Our results greatly improve and extend those of the authors whose related researches just involve a single mapping and the weaker $\Delta$-convergence. (C)2017 All rights reserved.
\end{abstract}

Keywords: Krasnoselski-Mann iteration, CAT(0) spaces, infinite families of nonexpansive mappings, strong convergence, $\Delta$-convergence.

2010 MSC: 47H09, 47H10, 47J25.

\section{Introduction}

Let $(X, d)$ be a metric space and $x, y \in X$ with $l=d(x, y)$. A geodesic path from $x$ to $y$ is an isometry $c:[0, l] \rightarrow X$ such that $c(0)=x$ and $c(l)=y$. The image of a geodesic path is called a geodesic segment, denoted by $[x, y]$ as it is unique. A metric space $X$ is a (uniquely) geodesic space if every two points of $X$ are joined by only one geodesic segment. A geodesic triangle $\triangle\left(x_{1}, x_{2}, x_{3}\right)$ in a geodesic space $X$ consists of three points $x_{1}, x_{2}, x_{3}$ of $X$ and three geodesic segments joining each pair of vertices. A comparison triangle of a geodesic triangle $\triangle\left(x_{1}, x_{2}, x_{3}\right)$ is the triangle $\bar{\triangle}\left(x_{1}, x_{2}, x_{3}\right):=\triangle\left(\bar{x}_{1}, \bar{x}_{2}, \bar{x}_{3}\right)$ in the Euclidean space $\mathbb{R}^{2}$ such that $d\left(x_{i}, x_{j}\right)=d_{\mathbb{R}^{2}}\left(\bar{x}_{i}, \bar{x}_{j}\right)$ for all $i, j=1,2,3$, where $\bar{x}_{i}$ is called the comparison vertex of $x_{i}, i=1,2,3$.

A geodesic space $X$ is a CAT(0) space if for each geodesic triangle $\triangle:=\triangle\left(x_{1}, x_{2}, x_{3}\right)$ in $X$ and its comparison triangle $\bar{\triangle}:=\triangle\left(\bar{x}_{1}, \bar{x}_{2}, \bar{x}_{3}\right)$ in $\mathbb{R}^{2}$, the CAT(0) inequality

$$
d(x, y) \leqslant d_{\mathbb{R}^{2}}(\bar{x}, \bar{y})
$$

is satisfied by all $x, y \in \triangle$ and their comparison points $\bar{x}, \bar{y} \in \bar{\triangle}$. The meaning of the $\operatorname{CAT}(0)$ inequality is that a geodesic triangle in $X$ is at least thin as its comparison triangle in the Euclidean plane. A thorough

\footnotetext{
*Corresponding author

Email addresses: qiansg1975@126.com (Shanguang Qian), dwq1273@126.com (Wei-Qi Deng)
} 
discussion of these spaces and their important role in various branches of mathematics are given in [1, 2]. The complex Hilbert ball with the hyperbolic metric is an example of a CAT(0) space (see [10]).

Fixed point theory in a CAT(0) space was first studied by Kirk (see $[13,15])$ who showed that every nonexpansive (single-valued) mapping defined on a bounded closed convex subset of a complete CAT $(0)$ space always has a fixed point. Since then the fixed point theory for single-valued and multivalued mappings in CAT(0) spaces has been rapidly developed and much papers have appeared (see, e.g., [3, 5$8,11,12,14,17,22-24])$.

In 2008, Kirk and Panyanak [16] used the concept of $\Delta$-convergence introduced by Lim [18] to prove the CAT(0) space analogs of some Banach space results which involve weak convergence, and Dhompongsa and Panyanak [9] obtained $\Delta$-convergence theorems for the Picard, Mann and Ishikawa iterations in the CAT $(0)$ space setting.

In 2010, Nanjaras and Panyanak [19] proved the demiclosed principle for asymptotically nonexpansive mappings in CAT(0) spaces. As a consequence, they also obtained a $\Delta$-convergence theorem of the Krasnoselski-Mann iteration for asymptotically nonexpansive mappings in this setting.

Inspired and motivated by those studies mentioned above, in this paper, by using a specific way of choosing the indices of the involved mappings and a new concept, namely, an analogue of inner product, we propose a modified Krasnoselski-Mann iteration scheme for approximating common fixed points of a countable family of asymptotically nonexpansive mappings and obtain a strong convergence theorem in CAT(0) space. The result improves and extends that of Nanjaras and Panyanak [19] whose related research involves just a single mapping and the weaker $\Delta$-convergence.

\section{Preliminaries}

In this paper, we write $(1-t) x \oplus t y$ for the the unique point $z$ in the geodesic segment joining from $x$ to $y$ such that

$$
d(z, x)=t d(x, y), d(z, y)=(1-t) d(x, y), \forall t \in[0,1]
$$

We also denote by $[x, y]$ the geodesic segment joining from $x$ to $y$, that is, $[x, y]:=\{(1-t) x \oplus t y: t \in[0,1]\}$. A subset $C$ of a $\operatorname{CAT}(0)$ space is convex if $[x, y] \subset C$ for all $x, y \in C$.

In the sequel we shall need the following preliminaries.

Let $X$ be a uniquely geodesic space equipped with two operations $\circ$ and $\oplus$, respectively defined by:

\section{Definition 2.1.}

(1) For any $\alpha \in \mathbb{R}$ and any $x \in X, \alpha \circ x$ stands for the unique point $u \in X$ such that

$$
\bar{u}=\alpha \bar{x},
$$

where ${ }^{-}$is the comparison vertex in the comparison triangle $\triangle(\bar{*}, \bar{\theta}, \bar{\cdot}):=\triangle(\bar{\bullet}, \overrightarrow{0}, \bar{\cdot})$ of $\triangle(\cdot, \theta, \cdot)$; and $\theta$ denotes a fixed $x_{0} \in X$.

(2) For any $x, y \in X, x \oplus y$ stands for the unique point $v \in X$ such that

$$
\bar{v}=\bar{x}+\bar{y},
$$

where $\bar{v}$ is the comparison vertex in the comparison triangles $\triangle(\bar{x}, \bar{\theta}, \bar{v})$ and $\triangle(\bar{y}, \bar{\theta}, \bar{v})$ of $\triangle(x, \theta, v)$ and $\triangle(y, \theta, v)$.

We then have the following conclusion:

Proposition 2.2. A uniquely geodesic space X equipped with two operations $\circ$ and $\oplus$ forms a vector space whenever its power is no larger than $\mathbf{X}$, namely, the cardinality of continuum. Such a space is called a geodesic vector space.

This follows from the fact that it is reasonable to define the mappings $x \mapsto \bar{x}$ and $v \mapsto \bar{v}$ as injections, determined respectively by the mappings $\triangle(x, \theta, x) \mapsto \triangle(\bar{x}, \bar{\theta}, \bar{x})$ and $(\triangle(x, \theta, v), \triangle(y, \theta, v)) \mapsto$ $(\triangle(\bar{x}, \bar{\theta}, \bar{v}), \triangle(\bar{y}, \bar{\theta}, \bar{v}))$, since $X$ is equivalent to $\mathbb{R}^{2}$. 
By the uniqueness of the negative element of any member of geodesic vector $X$, an operation $\ominus$ is defined by

$$
x \ominus y=x \oplus((-1) \circ y), \forall x, y \in X .
$$

Since a $\operatorname{CAT}(0)$ space is a uniquely geodesic space, then a $\operatorname{CAT}(0)$ space, equipped with two operations $\circ$ and $\oplus$, is called a $C A T(0)$ vector space whenever it possesses the cardinality of continuum.

Let $X$ be a CAT(0) vector space, with respect to which the following definition is given.

Definition 2.3. An analogue of inner product $\langle\cdot, \cdot\rangle: X \times X \rightarrow \mathbb{R}$ is defined by

$$
\langle x, y\rangle=\langle\bar{x}, \bar{y}\rangle_{\mathbb{R}^{2}}
$$

where $\bar{x}, \bar{y}$ are the comparison vertices in the comparison triangle $\triangle(\bar{x}, \bar{\theta}, \bar{y})$ of $\triangle(x, \theta, y)$.

It is obvious from the definition of the function $\langle\cdot, \cdot\rangle$ that it has the following properties: for any $x, y, z \in X$ and any $\alpha \in \mathbb{R}$,

(1) $\langle x, x\rangle \geqslant 0,\langle x, x\rangle=0 \Leftrightarrow x=\theta$;

(2) $\langle x, y\rangle=\langle y, x\rangle$;

(3) $\langle\alpha \circ x, y\rangle=\alpha\langle x, y\rangle$;

(4) $\langle x \oplus y, z\rangle=\langle x, z\rangle+\langle y, z\rangle$.

Then a distance $\rho$ on $X$ can be defined by

$$
\rho(x, y):=\sqrt{\langle x \ominus y, x \ominus y\rangle},
$$

which coincides with the original distance $d$ on $X$, since the distance $d_{\mathbb{R}^{2}}$ on $\mathbb{R}^{2}$ is just induced by $\langle\cdot, \cdot\rangle_{\mathbb{R}^{2}}$ and $d(x, y)=d_{\mathbb{R}^{2}}(\bar{x}, \bar{y})$.

Next, we define a function $\phi: X \times X \rightarrow \mathbb{R}^{+}$by

$$
\phi(x, y):=d^{2}(x, y),
$$

which obviously has the following property:

$$
\phi(y, x)=\phi(z, x)+\phi(y, z)+2\langle z \ominus y, x \ominus z\rangle, \forall x, y, z \in X
$$

Lemma 2.4 ([20]). Let $\left\{a_{n}\right\},\left\{\delta_{n}\right\}$, and $\left\{b_{n}\right\}$ be sequences of nonnegative real numbers satisfying

$$
a_{n+1} \leqslant\left(1+\delta_{n}\right) a_{n}+b_{n}, \forall n \in \mathbb{N} .
$$

If $\sum_{n=1}^{\infty} \delta_{n}<\infty$ and $\sum_{n=1}^{\infty} b_{n}<\infty$, then $\lim _{n \rightarrow \infty} a_{n}$ exists.

Lemma 2.5 ([21]). A geodesic space $X$ is a CAT(0) space if and only if the following inequality

$$
\mathrm{d}^{2}((1-\mathrm{t}) \mathrm{x} \oplus \mathrm{ty}, z) \leqslant(1-\mathrm{t}) \mathrm{d}^{2}(x, z)+\mathrm{td}^{2}(\mathrm{y}, z)-\mathrm{t}(1-\mathrm{t}) \mathrm{d}^{2}(\mathrm{x}, \mathrm{y})
$$

is satisfied by all $x, y, z \in X$ and all $t \in[0,1]$. In particular, if $x, y, z$ are points in a CAT $(0)$ space and $t \in[0,1]$, then

$$
d((1-t) x \oplus t y, z) \leqslant(1-t) d(x, z)+t d(y, z) .
$$

Let $\left\{x_{n}\right\}$ be a bounded sequence in a $\operatorname{CAT}(0)$ space $X$. For $x \in X$, we set

$$
r\left(x,\left\{x_{n}\right\}\right)=\limsup _{n \rightarrow \infty} d\left(x, x_{n}\right) .
$$

The asymptotic radius $\mathrm{r}\left(\left\{x_{n}\right\}\right)$ of $\left\{x_{n}\right\}$ is given by

$$
r\left(\left\{x_{n}\right\}\right)=\inf \left\{r\left(x,\left\{x_{n}\right\}\right): x \in K\right\}
$$

and the asymptotic center $\mathrm{A}\left(\left\{x_{n}\right\}\right)$ of $\left\{x_{n}\right\}$ is the set

$$
A\left(\left\{x_{n}\right\}\right)=\left\{x \in K: r\left(x,\left\{x_{n}\right\}\right)=r\left(\left\{x_{n}\right\}\right)\right\} .
$$

It is known (see, e.g., [8]) that in a $\operatorname{CAT}(0)$ space, $A\left(\left\{x_{n}\right\}\right)$ consists of exactly one point. We now give the definition of $\Delta$-convergence. 
Definition $2.6([16,18])$. A sequence $\left\{x_{n}\right\}$ in a $\operatorname{CAT}(0)$ space $X$ is said to $\Delta$-converge to $x \in X$ if $x$ is the unique asymptotic center of $\left\{u_{n}\right\}$ for every subsequence $\left\{u_{n}\right\}$ of $\left\{x_{n}\right\}$. In this case one writes $\Delta$ $\lim _{n \rightarrow \infty} x_{n}=x$ and calls $x$ the $\Delta$-limit of $\left\{x_{n}\right\}$.

Recall that a mapping $\mathrm{T}: \mathrm{C} \rightarrow \mathrm{C}$ is called asymptotically nonexpansive if there exists a sequence $\left\{\mu_{n}\right\} \subset[0, \infty)$ satisfying $\mu_{n} \rightarrow 0$ as $n \rightarrow \infty$ such that

$$
d\left(T^{n} x, T^{n} y\right) \leqslant\left(1+\mu_{n}\right) d(x, y), \forall x, y \in C, \forall n \in \mathbb{N} .
$$

Lemma 2.7 ([9]). Let $K$ be a closed convex subset of a complete CAT(0) space $X$, and let $\mathrm{T}: \mathrm{K} \rightarrow \mathrm{X}$ be a nonexpansive mapping. Suppose $\left\{x_{n}\right\}$ is a bounded sequence in $K$ such that $\lim _{n \rightarrow \infty} \mathrm{d}\left(\mathrm{x}_{n}, T x_{n}\right)=0$ and $\left\{\mathrm{d}\left(\mathrm{x}_{n}, v\right)\right\}$ converges for all $v \in F(T)$, then $\omega_{w}\left(x_{n}\right) \subset F(T)$. Here $\omega_{w}\left(x_{n}\right):=\cup A\left(\left\{x_{n}\right\}\right)$ where the union is taken over all subsequences $\left\{u_{n}\right\}$ of $\left\{x_{n}\right\}$. Moreover, $\omega_{w}\left(x_{n}\right)$ consists of exactly one point.

We now turn to a wider class of spaces, namely, the class of hyperbolic spaces, which contains the class of CAT(0) spaces (see Lemma 2.11).

Definition $2.8([17])$. A hyperbolic space is a triple $(X, d, W)$ where $(X, d)$ is a metric space and $W$ : $\mathrm{X} \times \mathrm{X} \times[0,1] \rightarrow \mathrm{X}$ is such that

(W1) $d(z, W(x, y, \alpha)) \leqslant(1-\alpha) d(z, x)+\alpha d(z, y)$;

(W2) $d(W(x, y, \alpha), W(x, y, \beta))=|\alpha-\beta| d(x, y)$;

(W3) $W(x, y, \alpha)=W(y, x, 1-\alpha)$;

(W4) $d(W(x, z, \alpha), W(y, w, \alpha))=(1-\alpha) d(x, y)+\alpha d(z, y)$ for all $x, y, z, w \in X, \alpha, \beta \in[0,1]$.

It follows from (W1) that for each $x, y \in X$ and $\alpha \in[0,1]$,

$$
d(x, W(x, y, \alpha)) \leqslant \alpha d(x, y), \quad d(y, W(x, y, \alpha)) \leqslant(1-\alpha) d(x, y) .
$$

Comparing (2.3) with (2.1), we can also use the notation $(1-\alpha) x \oplus \alpha y$ for $W(x, y, \alpha)$ in a hyperbolic space $(X, d, W)$.

Definition 2.9 ([17]). The hyperbolic space $(X, d, W)$ is called uniformly convex if for any $r>0$ and $\epsilon \in(0,2]$ there exists a $\delta \in(0,1]$ such that for all $a, x, y \in X$,

$$
\left.\begin{array}{l}
d(x, a) \leqslant r \\
d(y, a) \leqslant r \\
d(x, y) \leqslant \epsilon r
\end{array}\right\} \Rightarrow d\left(\frac{1}{2} x \oplus \frac{1}{2} y, a\right) \leqslant(1-\delta) r .
$$

A mapping $\eta:(0, \infty) \times(0,2] \rightarrow(0,1]$ providing such a $\delta:=\eta(r, \epsilon)$ for given $r>0$ and $\epsilon \in(0,2]$ is called a modulus of uniform convexity.

Lemma 2.10 ([17]). Let $(X, d, W)$ be a uniformly convex hyperbolic with modulus of uniform convexity $\eta$. For any $r>0, \epsilon \in(0,2], \lambda \in[0,1]$, and $a, x, y \in X$,

$$
\left.\begin{array}{l}
d(x, a) \leqslant r \\
d(y, a) \leqslant r \\
d(x, y) \leqslant \epsilon r
\end{array}\right\} \Rightarrow d((1-\lambda) x \oplus \lambda y, a) \leqslant(1-2 \lambda(1-\lambda) \eta(r, \epsilon)) r .
$$

Lemma 2.11 ([17]). Assume that $X$ is a CAT(0) space. Then $X$ is uniformly convex, and

$$
\eta(r, \epsilon)=\frac{\epsilon^{2}}{8}
$$

is a modulus of uniform convexity. 
Lemma 2.12 ([4]). The unique solutions to the positive integer equation

$$
n=i_{n}+\frac{\left(m_{n}-1\right) m_{n}}{2}, m_{n} \geqslant i_{n}, n=1,2,3, \ldots
$$

are

$$
i_{n}=n-\frac{\left(m_{n}-1\right) m_{n}}{2}, m_{n}=-\left[\frac{1}{2}-\sqrt{2 n+\frac{1}{4}}\right], n=1,2,3, \ldots,
$$

where $[x]$ denotes the maximal integer that is not larger than $x$.

\section{Main results}

Theorem 3.1. Let $\mathrm{X}$ be a complete $C A T(0)$ vector space and $C$ a closed convex nonempty subset of $X$. Let $\left\{\mathrm{T}_{i}\right\}_{i=1}^{\infty}$ : $\mathrm{C} \rightarrow \mathrm{C}$ be a sequence of nonexpansive mappings with a sequence $\left\{\mu_{n}^{(i)}\right\}$ satisfying $\sum_{i=1}^{\infty} \sum_{n=1}^{\infty} \mu_{n}^{(i)}<\infty$ and the interior of $F:=\cap_{i=1}^{\infty} F\left(T_{i}\right) \neq \emptyset$. Starting from an arbitrary $x_{1} \in C$, define $\left\{x_{n}\right\}$ by

$$
x_{n+1}=\left(1-\alpha_{n}\right) x_{n} \oplus \alpha_{n}\left(T_{n}^{*}\right)^{m_{n}} x_{n}, \forall n \in \mathbb{N},
$$

where $\left\{\alpha_{n}\right\} \subset[\epsilon, 1-\epsilon]$ for some $\epsilon>0$ and $\mathrm{T}_{n}^{*}=\mathrm{T}_{i_{n}}$ with $i_{n}$ and $\mathrm{m}_{n}$ being the solutions to the positive integer equation: $n=i_{n}+\frac{\left(m_{n}-1\right) m_{n}}{2}\left(m_{n} \geqslant i_{n}, n=1,2, \ldots\right)$, that is, for each $n \in \mathbb{N}$, there exist unique $i_{n}$ and $m_{n}$ such that

$$
\begin{aligned}
i_{1} & =1, i_{2}=1, i_{3}=2, i_{4}=1, i_{5}=2, i_{6}=3, i_{7}=1, i_{8}=2, \ldots \\
m_{1} & =1, m_{2}=2, m_{3}=2, m_{4}=3, m_{5}=3, m_{6}=3, m_{7}=4, m_{8}=4, \ldots
\end{aligned}
$$

Then $\left\{x_{n}\right\}$ converges strongly to a common fixed point $x^{*}$ of the mappings $\left\{\mathrm{T}_{i}\right\}_{i=1}^{\infty}$.

Proof. We divide the proof into several steps.

(I) $\lim _{n \rightarrow \infty} d\left(x_{n}, q\right)$ exists, $\forall q \in F$.

From (3.1), we have

$$
\begin{aligned}
d\left(x_{n+1}, q\right) & =d\left(\left(1-\alpha_{n}\right) x_{n} \oplus \alpha_{n}\left(T_{n}^{*}\right)^{m_{n}} x_{n}, q\right) \\
& \leqslant\left(1-\alpha_{n}\right) d\left(x_{n}, q\right)+\alpha_{n} d\left(\left(T_{n}^{*}\right)^{m_{n}} x_{n},\left(T_{n}^{*}\right)^{m_{n}} q\right) \\
& \leqslant\left(1-\alpha_{n}\right) d\left(x_{n}, q\right)+\alpha_{n}\left(1+\mu_{m_{n}}^{\left(i_{n}\right)}\right) d\left(x_{n}, q\right) \\
& \leqslant\left(1+\mu_{m_{n}}^{\left(i_{n}\right)}\right) d\left(x_{n}, q\right) .
\end{aligned}
$$

Note that $\sum_{n=1}^{\infty} \mu_{m_{n}}^{\left(i_{n}\right)}=\sum_{i=1}^{\infty} \sum_{n=i}^{\infty} \mu_{n}^{(i)} \leqslant \sum_{i=1}^{\infty} \sum_{n=1}^{\infty} \mu_{n}^{(i)}<\infty$. So by Lemma 2.4 we conclude $\lim _{n \rightarrow \infty} d\left(x_{n}, q\right)$ exists and hence $\left\{x_{n}\right\}$ and $\left\{\left(T_{n}^{*}\right)^{m_{n}} x_{n}\right\}$ are bounded.

(II) $x_{n} \rightarrow x^{*} \in \mathrm{C}$ as $\mathrm{n} \rightarrow \infty$.

For any $\mathrm{q} \in \mathrm{F}$, we have, by Lemma 2.5,

$$
\begin{aligned}
d^{2}\left(x_{n+1}, q\right) & =d^{2}\left(\left(1-\alpha_{n}\right) x_{n} \oplus \alpha_{n}\left(T_{n}^{*}\right)^{m_{n}} x_{n}, q\right) \\
& \leqslant\left(1-\alpha_{n}\right) d^{2}\left(x_{n}, q\right)+\alpha_{n} d^{2}\left(\left(T_{n}^{*}\right)^{m_{n}} x_{n}, q\right)-\alpha_{n}\left(1-\alpha_{n}\right) d^{2}\left(x_{n},\left(T_{n}^{*}\right)^{m_{n}} x_{n}\right) \\
& \leqslant\left(1-\alpha_{n}\right) d^{2}\left(x_{n}, q\right)+\alpha_{n}\left(1+\mu_{m_{n}}^{\left(i_{n}\right)}\right) d^{2}\left(x_{n}, q\right)-\alpha_{n}\left(1-\alpha_{n}\right) d^{2}\left(x_{n},\left(T_{n}^{*}\right)^{m_{n}} x_{n}\right) \\
& \leqslant\left(1+\mu_{m_{n}}^{\left(i_{n}\right)}\right) d^{2}\left(x_{n}, q\right) \\
& =d^{2}\left(x_{n}, q\right)+v_{m_{n}}^{\left(i_{n}\right)}
\end{aligned}
$$


where $v_{m_{n}}^{\left(i_{n}\right)}:=\mu_{m_{n}}^{\left(i_{n}\right)} d^{2}\left(x_{n}, q\right)$, and so $\sum_{n=1}^{\infty} v_{m_{n}}^{\left(i_{n}\right)}<\infty$. Furthermore, it follows from (2.2) that

$$
\phi\left(p, x_{n}\right)=\phi\left(x_{n+1}, x_{n}\right)+\phi\left(p, x_{n+1}\right)+2\left\langle x_{n+1} \ominus p, x_{n} \ominus x_{n+1}\right\rangle, \forall p \in X .
$$

This implies that

$$
\left\langle x_{n+1} \ominus p, x_{n} \ominus x_{n+1}\right\rangle+\frac{1}{2} \phi\left(x_{n+1}, x_{n}\right)=\frac{1}{2}\left(\phi\left(p, x_{n}\right)-\phi\left(p, x_{n+1}\right)\right) .
$$

Moreover, since the interior of $F$ is nonempty, there exists a $p^{*} \in F$ and $r>0$ such that $\left(p^{*} \oplus r \circ h\right) \in F$ whenever $\sqrt{\langle h, h\rangle} \leqslant 1$. Thus, from (3.2) and (3.3) we obtain

$$
0 \leqslant\left\langle x_{n+1} \ominus\left(p^{*} \oplus r \circ h\right), x_{n} \ominus x_{n+1}\right\rangle+\frac{1}{2} \phi\left(x_{n+1}, x_{n}\right)+\frac{1}{2} v_{m_{n}}^{\left(i_{n}\right)} .
$$

Then from (3.3) and (3.4) we obtain

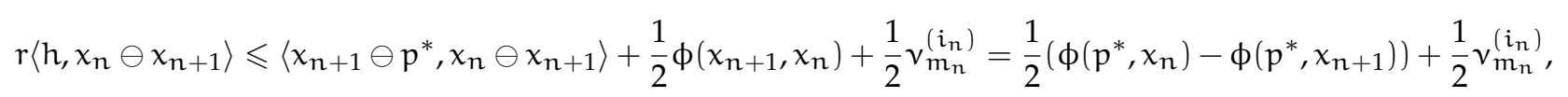

and hence

$$
\left\langle h, x_{n} \ominus x_{n+1}\right\rangle \leqslant \frac{1}{2 r}\left(\phi\left(p^{*}, x_{n}\right)-\phi\left(p^{*}, x_{n+1}\right)\right)+\frac{1}{2 r} v_{m_{n}}^{\left(i_{n}\right)} .
$$

Since $h$ with $\sqrt{\langle h, h\rangle} \leqslant 1$ is arbitrary, we have, by taking $h=\frac{1}{d\left(x_{n}, x_{n+1}\right)} \circ\left(x_{n} \ominus x_{n+1}\right)$,

$$
d\left(x_{n}, x_{n+1}\right) \leqslant \frac{1}{2 r}\left(\phi\left(p^{*}, x_{n}\right)-\phi\left(p^{*}, x_{n+1}\right)\right)+\frac{1}{2 r} v_{m_{n}}^{\left(i_{n}\right)}
$$

So, if $n>m$, then we have

$$
\begin{aligned}
d\left(x_{m}, x_{n}\right) \leqslant \sum_{j=m}^{n-1} d\left(x_{j}, x_{j+1}\right) & \leqslant \frac{1}{2 r} \sum_{j=m}^{n-1}\left(\phi\left(p^{*}, x_{j}\right)-\phi\left(p^{*}, x_{j+1}\right)\right)+\frac{1}{2 r} \sum_{j=m}^{n-1} v_{m_{j}}^{\left(i_{j}\right)} \\
& =\frac{1}{2 r}\left(\phi\left(p^{*}, x_{m}\right)-\phi\left(p^{*}, x_{n}\right)\right)+\frac{1}{2 r} \sum_{j=m}^{n-1} v_{m_{j}}^{\left(i_{j}\right)} .
\end{aligned}
$$

But we know that $\left\{\phi\left(p^{*}, x_{n}\right)\right\}$ converges, and $\sum_{n=1}^{\infty} v_{m_{n}}^{\left(i_{n}\right)}<\infty$. Therefore, we obtain from (3.6) that $\left\{x_{n}\right\}$ is a Cauchy sequence. Since $X$ is complete there exists an $x^{*} \in X$ such that $x_{n} \rightarrow x^{*} \in X$ as $n \rightarrow \infty$. Thus, since $\left\{x_{n}\right\} \subset C$ and $C$ is closed and convex, then $x^{*} \in C$, that is,

$$
x_{n} \rightarrow x^{*} \in C(n \rightarrow \infty) \text {. }
$$

(III) $x^{*}$ is one of members of $\mathrm{F}$.

Since $\left\{\alpha_{n}\right\} \subset[\epsilon, 1-\epsilon]$, we have, from (3.2),

$$
\epsilon^{2} d^{2}\left(x_{n},\left(T_{n}^{*}\right)^{m_{n}} x_{n}\right) \leqslant d^{2}\left(x_{n}, q\right)-d^{2}\left(x_{n+1}, q\right)+v_{m_{n}}^{\left(i_{n}\right)}
$$

so that

$$
\epsilon^{2} \sum_{n=1}^{\infty} d^{2}\left(x_{n},\left(T_{n}^{*}\right)^{m_{n}} x_{n}\right) \leqslant d^{2}\left(x_{1}, q\right)+\sum_{n=1}^{\infty} v_{m_{n}}^{\left(i_{n}\right)}<\infty
$$

This implies that

$$
\lim _{n \rightarrow \infty} d\left(x_{n},\left(T_{n}^{*}\right)^{m_{n}} x_{n}\right)=0
$$

It follows from (3.5) that

$$
\lim _{n \rightarrow \infty} d\left(x_{n+1}, x_{n}\right)=0
$$


which implies that, by induction, for any nonnegative integer $j$,

$$
\lim _{n \rightarrow \infty} d\left(x_{n+j}, x_{n}\right)=0 \text {. }
$$

Next, for any $i \in \mathbb{N}$, we consider the corresponding subsequence $\left\{x_{k}^{(i)}\right\}_{k \in \Gamma_{i}}$ of $\left\{x_{n}\right\}$, where $k \in \Gamma_{i}:=$ $\left\{k \in \mathbb{N}: k=i_{k}+\frac{\left(j_{k}-1\right) j_{k}}{2}, j_{k} \geqslant i_{k}, j_{k} \in \mathbb{N}\right\}$. For example, by Lemma 2.12 and the definition of $\Gamma_{1}$, we have $\Gamma_{1}=\{1,2,4,7,11,16, \ldots\}$ and $\mathfrak{i}_{1}=\mathfrak{i}_{2}=\mathfrak{i}_{4}=\mathfrak{i}_{7}=\mathfrak{i}_{11}=\mathfrak{i}_{16}=\cdots=1$. For simplicity, $\left\{x_{k}^{(i)}\right\}_{k \in \Gamma_{i}}$, $\left\{\left(T_{k}^{*}\right)^{(i)}\right\}_{k \in \Gamma_{i}}$, and $\left\{j_{k}^{(i)}\right\}_{k \in \Gamma_{i}}$ are written as $\left\{x_{n}^{\prime}\right\},\left\{T_{n}^{\prime}\right\}$, and $\left\{m_{n}\right\}$, respectively. Since $m_{n} \geqslant 2$ whenever $n \geqslant 2$, we have, for each $n \in \mathbb{N}$,

$$
\begin{aligned}
d\left(x_{n}^{\prime}, T_{n}^{\prime} x_{n}^{\prime}\right) \leqslant & d\left(x_{n+1}^{\prime}, x_{n}^{\prime}\right)+d\left(x_{n+1}^{\prime},\left(T_{n+1}^{\prime}\right)^{m_{n+1}} x_{n+1}^{\prime}\right) \\
& +d\left(\left(T_{n+1}^{\prime}\right)^{m_{n+1}} x_{n+1}^{\prime},\left(T_{n+1}^{\prime}\right)^{m_{n+1}} x_{n}^{\prime}\right)+d\left(\left(T_{n+1}^{\prime}\right)^{m_{n+1}} x_{n}^{\prime}, T_{n}^{\prime} x_{n}^{\prime}\right) \\
\leqslant & d\left(x_{n+1}^{\prime}, x_{n}^{\prime}\right)+d\left(x_{n+1}^{\prime},\left(T_{n+1}^{\prime}\right)^{m_{n+1}} x_{n+1}^{\prime}\right) \\
& +\left(1+\mu_{m_{n+1}}^{(i)}\right) d\left(x_{n+1}^{\prime}, x_{n}^{\prime}\right)+d\left(\left(T_{n+1}^{\prime}\right)^{m_{n+1}} x_{n}^{\prime}, T_{n}^{\prime} x_{n}^{\prime}\right) .
\end{aligned}
$$

Note that $\left\{m_{n}\right\}_{n \in \Gamma_{i}}=\{i, i+1, i+2, \ldots\}$, i.e., $m_{n+1}-1=m_{n}, \mu_{1}^{\left(i_{k}\right)}=\mu_{1}^{(i)}$, and $T_{n}^{\prime}=T_{i}=T_{n+1}^{\prime}$ whenever $k \in \Gamma_{i}$. Then from (3.8), we have, as $n \rightarrow \infty$,

$$
\begin{aligned}
d\left(\left(T_{n+1}^{\prime}\right)^{m_{n+1}} x_{n}^{\prime}, T_{n}^{\prime} x_{n}^{\prime}\right) & =d\left(\left(T_{n+1}^{\prime}\right)\left(\left(T_{n+1}^{\prime}\right)^{m_{n+1}-1} x_{n}^{\prime}\right), T_{n}^{\prime} x_{n}^{\prime}\right) \\
& \leqslant\left(1+\mu_{1}^{(i)}\right) d\left(\left(T_{n+1}^{\prime}\right)^{m_{n+1}-1} x_{n}^{\prime}, x_{n}^{\prime}\right) \\
& =\left(1+\mu_{1}^{(i)}\right) d\left(\left(T_{n}^{\prime}\right)^{m_{n}} x_{n}^{\prime}, x_{n}^{\prime}\right) \rightarrow 0 .
\end{aligned}
$$

Then it follows from (3.8) and (3.9) that

$$
\lim _{n \rightarrow \infty} d\left(x_{n}^{\prime}, T_{n}^{\prime} x_{n}^{\prime}\right)=0
$$

That is, for each $i \in \mathbb{N}$, there exists a subsequence $\left\{x_{n}^{(i)}\right\}$ of $\left\{x_{n}\right\}$ such that

$$
\lim _{n \rightarrow \infty} d\left(x_{n}^{(i)},\left(\left(T_{n}^{*}\right)^{(i)} x_{n}^{(i)}\right)=0\right.
$$

Since $\left(T_{n}^{*}\right)^{(i)}=T_{i}$, we have, for each $i \in \mathbb{N}$,

$$
\lim _{n \rightarrow \infty} d\left(x_{n}^{(i)}, T_{i} x_{n}^{(i)}\right)=0
$$

Thus, from (3.7), since for any $i \in \mathbb{N}, x_{n}^{(i)} \rightarrow x^{*}$ as $n \rightarrow \infty$ and $T_{i}$ is continuous, we obtain $x^{*} \in F\left(T_{i}\right)$, i.e., $x^{*} \in \cap_{i=1}^{\infty} F\left(T_{i}\right)$. The proof is completed.

\section{Acknowledgment}

The authors wish to thank the anonymous referees for their careful reading of the manuscript. This study is supported by the General Science Foundation of Yunnan province education department (2015Y280).

\section{References}

[1] M. R. Bridson, A. Haefliger, Metric spaces of non-positive curvature, Grundlehren der Mathematischen Wissenschaften [Fundamental Principles of Mathematical Sciences], Springer-Verlag, Berlin, (1999). 1

[2] D. Burago, Y. Burago, S. Ivanov, A course in metric geometry, Graduate Studies in Mathematics, American Mathematical Society, Providence, RI, (2001). 1

[3] P. Chaoha, A. Phon-on, A note on fixed point sets in CAT(0) spaces, J. Math. Anal. Appl., 320 (2006), 983-987.1 
[4] W.-Q. Deng, P. Bai, An implicit iteration process for common fixed points of two infinite families of asymptotically nonexpansive mappings in Banach spaces, J. Appl. Math., 2013 (2013), 6 pages. 2.12

[5] S. Dhompongsa, W. Fupinwong, A. Kaewkhao, Common fixed points of a nonexpansive semigroup and a convergence theorem for Mann iterations in geodesic metric spaces, Nonlinear Anal., 70 (2009), 4268-4273. 1

[6] S. Dhompongsa, A. Kaewkhao, B. Panyanak, Lim's theorems for multivalued mappings in CAT(0) spaces, J. Math. Anal. Appl., 312 (2005), 478-487.

[7] S. Dhompongsa, W. A. Kirk, B. Panyanak, Nonexpansive set-valued mappings in metric and Banach spaces, J. Nonlinear Convex Anal., 8 (2007), 35-45.

[8] S. Dhompongsa, W. A. Kirk, B. Sims, Fixed points of uniformly Lipschitzian mappings, Nonlinear Anal., 65 (2006), 762-772. 1, 2

[9] S. Dhompongsa, B. Panyanak, On $\Delta$-convergence theorems in CAT(0) spaces, Comput. Appl. Math., 56 (2008), 25722579. 1, 2.7

[10] K. Goebel, S. Reich, Uniform convexity, hyperbolic geometry, and nonexpansive mappings, Monographs and Textbooks in Pure and Applied Mathematics, Marcel Dekker, Inc., New York, (1984). 1

[11] N. Hussain, M. A. Khamsi, On asymptotic pointwise contractions in metric spaces, Nonlinear Anal., 71 (2009), $4423-$ 4429. 1

[12] A. Kaewcharoen, W. A. Kirk, Proximinality in geodesic spaces, Abstr. Appl. Anal., 2006 (2006), 10 pages. 1

[13] W. A. Kirk, Geodesic geometry and fixed point theory, Seminar of Mathematical Analysis, Malaga/Seville, (2002/2003), Colecc. Abierta, Univ. Sevilla Secr. Publ., Seville, 64 (2003), 195-225. 1

[14] W. A. Kirk, Fixed point theorems in CAT(0) spaces and $\mathbb{R}$-trees, Fixed Point Theory Appl., 2004 (2004), 8 pages. 1

[15] W. A. Kirk, Geodesic geometry and fixed point theory, II, International Conference on Fixed Point Theory and Applications Yokohama Publ., Yokohama, (2004), 113-142. 1

[16] W. A. Kirk, B. Panyanak, A concept of convergence in geodesic spaces, Nonlinear Anal., 68 (2008), 3689-3696. 1, 2.6

[17] L. Leustean, A quadratic rate of asymptotic regularity for CAT(0)-spaces, J. Math. Anal. Appl., 325 (2007), 386-399. 1, $2.8,2.9,2.10,2.11$

[18] T. C. Lim, Remarks on some fixed point theorems, Proc. Amer. Math. Soc., 60 (1976), 179-182. 1, 2.6

[19] B. Nanjaras, B. Panyanak, Demiclosed principle for asymptotically nonexpansive mappings in CAT(0) spaces, Fixed Point Theory Appl., 2010 (2010), 14 pages. 1

[20] M. O. Osilike, S. C. Aniagbosor, B. G. Akuchu, Fixed points of asymptotically demicontractive mappings in arbitrary Banach spaces, PanAmer. Math. J., 12 (2002), 77-88. 2.4

[21] S. Saejung, Halpern's iteration in CAT(0) spaces, Fixed Point Theory Appl., 2010 (2010), 13 pages. 2.5

[22] N. Shahzad, Fixed point results for multimaps in CAT(0) spaces, Topology Appl., 156 (2009), 997-1001. 1

[23] N. Shahzad, Invariant approximations in CAT(0) spaces, Nonlinear Anal., 70 (2009), 4338-4340.

[24] N. Shahzad, J. Markin, Invariant approximations for commuting mappings in CAT $(0)$ and hyperconvex spaces, J. Math. Anal. Appl., 337 (2008), 1457-1464. 1 